\title{
EDITORIAL
}

\section{IPF or NSIP? That is the question}

\author{
C. Vogelmeier
}

Bronchoalveolar lavage (BAL) has been crucial in elucidating the immune effector cells involved in the inflammatory processes in idiopathic interstitial pneumonias [1]. Despite its value as a research tool, the diagnostic usefulness of BAL in idiopathic interstitial pneumonias is limited. An increase in neutrophils and/or eosinophils and/or lymphocytes may be seen. The idiopathic interstitial pneumonia with the worst prognosis is idiopathic pulmonary fibrosis (IPF) showing a histological pattern of usual interstitial pneumonia (UIP) [2]. In IPF, historical data suggests that certain changes in BAL cell differentials may have prognostic importance; in some studies, patients with increased percentages of neutrophils or eosinophils (or both) had a worse prognosis, whereas BAL lymphocytosis has been associated with a greater responsiveness to corticosteroid therapy [3-6].

The most detailed data in that context have been published by WATTERS et al. [4]. In their cohort, pretreatment BAL lymphocytosis was associated with significant subsequent clinical improvement. They also reported on the histology of their patients, evaluating 11 separate histological features. They found that BAL lymphcytosis was associated with moderate-to-severe alveolar septal inflammation and with a relative lack of histological honeycombing. These data suggested that BAL fluid lymphocytosis identifies a subset of patients with IPF who are more "cellular" in biopsy and likely to improve in response to corticosteroid therapy.

KATZENSTEIN and MYERS [7] already emphasised the limitations of this study. Only 19 patients were available for follow-up at $1 \mathrm{yr}$. Of these, five of seven with BAL lymphocytosis improved, compared with none of 12 patients without BAL lymphocytosis, although nine of the latter remained stable and only three deteriorated. Besides, the question is appropriate, if in the light of the new idiopathic interstitial pneumonia classification [2] these patients with BAL lymphocytosis really suffered from IPF.

Importantly, several studies showed a marked BAL lymphocytosis in patients with nonspecific interstitial pneumonia (NSIP). NSIP includes a wide variety of pathological features. Histology may be dominated by interstitial inflammation or fibrosis or a combination of both. Some patients with NSIP recover completely, most stabilise or improve on treatment. It was suggested that the patients (formerly suspected of suffering from IPF) with BAL lymphocytosis in reality had some form of NSIP [2]. In the near future, a separation of NSIP into four clinico-radiological syndromes will be proposed: 1) an IPF variant; 2) a scarring form of organising pneumonia; 3) NSIP in extrinsic allergic alveolitis; and 4) NSIP in connective tissue disorders. Of these, only the IPF variant may cause diagnostic problems and thus patients may be misclassified as having IPF. As mentioned before, the

Correspondence: C. Vogelmeier, Division for Pulmonary Disorders, Dept of Internal Medicine, Philipps-University Marburg, Baldingerstrasse, 35033 Marburg, Germany. Fax: 49 64212868987. E-mail: Claus.Vogelmeier@med.uni-marburg.de differentiation is important as idiopathic NSIP has an overall better prognosis than UIP.

The differential diagnosis between IPF and the IPF variant of NSIP may be demanding as they present with similar or identical clinical pictures. The gold standard for getting to the right diagnosis is surgical lung biopsy. Nevertheless, in most clinical series describing patients with presumed IPF, surgical lung biopsies were only performed in a minority of patients [1]. In contrast, BAL is widely used in the diagnostic work-up of patients with idiopathic interstitial pneumonias. Thus, the question arises if BAL may be helpful to differentiate between IPF and the IPF variant of NSIP.

This issue of the European Respiratory Journal contains a study by VeERARAgHAVAN et al. [8]. They hypothesised that BAL findings may distinguish between IPF and the IPF variant of NSIP. In addition, the authors evaluated the prognostic value of BAL cell differentials within the disease subgroups. They retrospectively studied BAL findings in 54 patients with IPF $(n=35)$ and fibrotic NSIP $(n=19)$. Diagnosis was made in all patients by surgical lung biopsy, and UIP and NSIP subtypes were defined using the new American Thoracic Society (ATS)/European Respiratory Society criteria [2]. All patients had a clinical diagnosis of IPF according to the following criteria: bilateral, predominantly basal rales, restrictive functional defect or isolated reduction in the transfer factor of the lungs to carbon monoxide, chest radiographical abnormalities of pulmonary fibrosis in a distribution compatible with IPF, absence of significant occupational or environmental exposure or connective tissue disease. The major findings of the study are that the BAL total and differential cell counts do not differ between the two groups, and in neither group are BAL findings predictive of survival or changes in lung function at $1 \mathrm{yr}$, even after adjustment for disease severity, smoking and treatment. FLAHERTY et al. [9] have recently shown that the histological pattern of NSIP and UIP may be found in the same lung. To assess whether this occurrence could have confounded their results, VEERARAGHAVAN et al. [8] repeated the comparison in a cohort of 21 patients in whom the histological diagnosis was confirmed in biopsies from more than one lobe. Again there was no statistical difference in the total cell count and the cell differentials.

Based on these findings, the good outcome of the abovementioned historical series of IPF, associated with a BAL lympocytosis [4-6], cannot be ascribed to better survival in fibrotic NSIP. It is hard to tell what the diagnosis was in these patients from the current viewpoint; not all patients were diagnosed histologically. Furthermore, even in biopsied patients the appearances of cellular NSIP or desquamative interstitial pneumonia and respiratory bronchiolitis-associated interstitial lung disease would have been regarded as part of the spectrum of IPF at the time of the studies.

So, how is a differentiation between IPF and the IPF variant of NSIP made when a surgical lung biopsy is unobtainable? High-resolution computed tomography (HRCT) may be the answer. In patients with the IPF variant of NSIP, the HRCT findings consist of a spectrum of predominantly subpleural 
ground glass and reticular abnormalities without or with little honeycombing. Thus, the distribution of the abnormalities (subpleural) is similar in IPF and the IPF variant of NSIP, whereas the degree of ground-glass attenuation (NSIP $>$ IPF) and the extent of honeycombing (IPF $>$ NSIP) is different [2, 10]. Why did VEERARAGHAVAN et al. [8] not include HRCT in their study? They needed more than two decades (1978-2000) to collect their patients. As a consequence, a big portion of the patients belonged to the pre-computed tomography era.

What is left now for BAL in the diagnostic work-up of patients with diffuse parenchymal lung disorders? At first sight, the presented data add to the existing doubts on whether BAL cell counts should be performed routinely [11]. Nevertheless, the fact remains that the majority of patients with these disorders do not get surgical lung biopsies. Researchers may complain about this or try to get around the problem by adding up findings from non- or less-invasive methods, as suggested in the ATS guidelines [1]; the clinical history, the physical examination, the lung function changes and the laboratory findings may lead in certain directions.

Bronchoalveolar lavage (and transbronchial biopsies) may help to narrow the number of possible disorders or even substantiate a variety of specific diagnoses, e.g. malignancy, infections, eosinophilic pneumonia, pulmonary histiocytosis $\mathrm{X}$, alveolar proteinosis, alveolar haemorrhage, sarcoidosis and hypersensitivity pneumonitis. Together with high-resolution computed tomography, the right diagnosis should be achievable in the majority of patients. Thus, bronchoalveolar lavage by itself is not as good as was hoped, but it is a useful instrument in the orchestra of non-/less-invasive diagnostic methods.

\section{References}

1. American Thoracic Society. Idiopathic pulmonary fibrosis: diagnosis and treatment. International consensus statement. American Thoracic Society (ATS), and the European
Respiratory Society (ERS). Am J Respir Crit Care Med 2000; 161: 646-664.

2. American Thoracic Society/European Respiratory Society. International multidisciplinary consensus classification of the idiopathic interstitial pneumonias. Am J Respir Crit Care Med 2002; 165: 277-304.

3. Turner-Warwick M, Haslam PL. The value of serial bronchoalveolar lavages in assessing the clinical progress of patients with cryptogenic fibrosing alveolitis. Am Rev Respir Dis 1987; 135: 26-34.

4. Watters LC, Schwarz MI, Cherniack RM, et al. Idiopathic pulmonary fibrosis. Pretreatment bronchoalveolar lavage cellular constituents and their relationships with lung histopathology and clinical response to therapy. Am Rev Respir Dis 1987; 135: 696-704.

5. Rudd RM, Haslam PL, Turner-Warwick M. Cryptogenic fibrosing alveolitis. Relationships of pulmonary physiology and bronchoalveolar response to treatment and prognosis. Am Rev Respir Dis 1981; 124: 1-8.

6. Haslam PL, Turton CW, Lukoszek A, et al. Bronchoalveolar lavage fluid cell counts in cryptogenic fibrosing alveolitis and their relation to therapy. Thorax 1980; 35: 328-339.

7. Katzenstein A-LA, Myers JL. Idiopathic pulmonary fibrosis. Clinical relevance of pathologic classification. Am J Respir Crit Care Med 1998; 157: 1301-1315.

8. Veeraraghavan S, Latsi PI, Wells AU, et al. BAL findings in idiopathic nonspecific interstitial pneumonia and usual interstitial pneumonia. Eur Respir J 2003; 22: 239-244.

9. Flaherty KR, Travis WD, Colby TV, et al. Histopathologic variability in usual and nonspecific interstitial pneumonia. Am J Respir Crit Care Med 2001; 164: 1722-1727.

10. MacDonald SLS, Rubens MB, Hansell DM, et al. Nonspecific interstitial pneumonia and usual interstitial pneumonia: comparative appearances and diagnostic accuracy of high-resolution computed tomography. Radiology 2001; 221: 600-605.

11. British Thoracic Society. Recommendations. The diagnosis, assessment and treatment of diffuse parenchymal lung disease in adults. Thorax 1999; 54: S1-S30. 